Correspondence: S Nainiwal,

House No. B-297, Harimarg,

Malviya-Nagar,

Jaipur - 302017

Rajasthan, India

Tel: + 91-141-2521540;

Fax: + 91-11-26588919.

E-mail: nainvision@yahoo.com

Eye (2005) 19, 1012-1013. doi:10.1038/sj.eye.6701704; published online 12 November 2004

Sir,

\section{Uveitis and the menstrual cycle}

We read with interest the article 'Uveitis and the menstrual cycle' by Sanghvi et al. ${ }^{1}$ In their study, the authors reported the relation between menstrual cycle and patient-reported onset of acute anterior uveitis (AAU), in 76 regularly menstruating women. They concluded that the onset of AAU was partially dependent on the levels of either oestrogen or progesterone, or both. They summarized that they have demonstrated a significant increase in the incidence of AAU arising late in the menstrual period. While we applaud the authors' efforts on this important issue, we felt that the authors have to clarify a few points before arriving at their conclusion.

First, the authors admitted that they have not performed any serum assay of hormones to demonstrate the relation between the onset of AAU and blood levels of oestrogen or progesterone. What they have shown in fact was a possible increase in incidence of AAU in late menstrual cycle. The authors considered that the incidence was significantly increased. However, as they have pointed out, in fact that was 'just approaching statistical significance but not yet achieving it'. What is more, while the recall bias for the date of commencement of last menstrual period (LMP) was probably not great, that for the date of commencement of onset of uveitis was yet unknown. It is not unusual for mild anterior uveitis to be relatively silent and asymptomatic in the early phase of the disease. The relationship between menstrual cycle and onset of AAU that the authors presented was thus better described as a possible one.

Second, anterior uveitis is a heterogeneous group of diseases. It would be of value to know the aetiologies of anterior uveitis in this group of patients, and especially in those recurrent cases. Anterior uveitis is known to be much more subclinical when associated with certain aetiologies, such as juvenile rheumatoid arthritis ${ }^{2}$ and inflammatory bowel disease. ${ }^{3}$ That again has bearing as to the accuracy of self-reported onset of AAU. It would also be interesting to see whether there are specific aetiologies that are particularly dependent on menstrual cycle.

Third, 65 out of 76 patients were having a second or subsequent attack. It would be imperative to know whether they were 'acute on chronic' cases in which they might still be put on low tailing down dose of steroid before the attack, which might modify their selfperceived onset of recurrent AAU.

We suggest that the authors may also try to see whether the acute uveitic attacks were particularly severe with respect to, for example, intraocular pressure rise, frequency and dosage of steroid required to abort the attack, etc., during a particular phase of menstrual cycle. We believe that reliance on objective signs may be a better measure than self-reported onset of disease.

\section{References}

1 Sanghvi C, Aziz K, Jones NP. Uveitis and the menstrual cycle. Eye 2004; 18: 451-454.

2 Kotaniemi K, Savolainen A, Karma A, Aho K. Recent advances in uveitis of juvenile idiopathic arthritis. Surv Ophthalmol 2003; 48(5): 489-502.

3 Rychwalski PJ, Cruz OA, Alanis-Lambreton G, Foy TM, Kane RE. Asymptomatic uveitis in young people with inflammatory bowel disease. J AAPOS 1997; 1(2): 111-114.

DYL Leung and DSC Lam

Department of Ophthalmology and Visual

Sciences, The Chinese University of Hong Kong 2/F.

Hong Kong Eye Hospital, 147K Argyle Street

Kowloon, Hong Kong, People's Republic of China

Correspondence: DYL Leung

Tel: $+852-27623000$

Fax: + 852-27687058

E-mail: dexleung@alumni.cuhk.net

Financial or proprietary interest: nil

Financial support: nil

Eye (2005) 19, 1013. doi:10.1038/sj.eye.6701707;

published online 24 September 2004

Sir,

\section{Female sex hormones and uveitis}

I read with interest the paper by Sanghvi et al ${ }^{1}$ 'Uveitis and the Menstrual Cycle'. The authors studied a group of 
patients that presented with new or recurrent episodes of acute anterior uveitis to determine the relationship between their incidences and the menstrual cycle. They found an increased incidence of these episodes during the postovulatory phase of the menstrual cycle. I have to commend the authors for their effort and their valuable and genuinely interesting findings. I have, however, a query and some comments to make.

The authors included patients on combined oral contraceptives and excluded those with irregular menstrual cycles and those who are postmenopausal, pregnant, or lactating. It was not mentioned, however, whether any of their patients were on immunomodulatory medications such as corticosteroids or other immunosuppressive or cytotoxic drugs for coexistent autoimmune diseases or if these patients were also excluded. Those on intermittent or on-demand therapy such as asthma sufferers were particularly important group to exclude since they tend to have their episodes in the premenstrual period, as quoted in the paper, and the administration of steroids around that period could certainly have affected the distribution of the incidence of their uveitis episodes.

The results, however, were extremely interesting. The authors found a significant increase in the incidence of acute anterior uveitis during the postovulatory phase and premenstrual period of the menstrual cycle. They postulated that this is mainly related to the rapid withdrawal of the protective anti-inflammatory effect of either oestrogen or progesterone or both in the late luteal phase. Although I agree with the authors that it is difficult to decide which hormone might be responsible, I would like to suggest that this anti-inflammatory effect is most probably related to the influence of oestrogen rather than progesterone, especially as the majority of the study patients experienced the inflammatory episodes during the late postovulatory phase of the cycle when normally oestrogen drops and progesterone peaks. Also, Salem $^{2}$ recently reviewed the role of oestrogen in immune response and demonstrated that several studies have provided evidence to the modulatory effect of oestrogen on the CD4-T cells subpopulation designated TH1 and TH2. Oestrogen was found to inhibit the production of TH1 proinflammatory cytokines such as IL-12, TNF- $\alpha$, and IFN- $\gamma$ but to stimulate the production of TH2 anti-inflammatory cytokines such as IL-10, IL-4, and TGF- $\beta$. Both increased aqueous IL- $12^{3}$ and decreased IL- $10^{4}$ have been linked to the development and severity of anterior uveitis and increased serum IFN- $\gamma$ has been associated with serious visual loss. ${ }^{5}$

The interesting findings of the study are relevant to a great number of female patients suffering from uveitis and point forward to several potential areas of research.
One can hypothesise that some of these patients who suffer incessant recurrences might have relatively lower oestrogen or progesterone levels that, perhaps, render them more vulnerable to recurrent inflammation. A possible beneficial effect would then be achieved in these patients by prescribing them, unless contraindicated, some form of hormonal supplement. Also, although the study excluded postmenopausal patients, it can be extrapolated that hormonal replacement therapy may be a useful therapeutic addition in these patients, especially in cases with recalcitrant and potentially sight-threatening recurrences. These hormones have been suggested to have anti-inflammatory effect through reducing E-selectin, ${ }^{6}$ a proinflammatory mediator involved in some types of uveitis. ${ }^{7,8}$ I am sure many would agree that some patients would be better off taking these hormones, if at all possible, instead of immunosuppressive or cytotoxic drugs. Only future studies can prove or refute these hypotheses.

\section{References}

1 Sanghvi C, Aziz K, Jones NP. Uveitis and the menstrual cycle. Eye 2004; 18: 451-454.

2 Salem ML. Estrogen, a double-edged sword: modulation of TH1- and TH2-mediated inflammations by differential regulation of TH1/TH2 cytokine production. Curr Drug Targets Inflamm Allerg 2004; 3: 97-104.

3 el Shabrawi Y, Livir-Rallatos C, Christen W, Baltatzis S, Foster CS. High levels of interleukin-12 in the aqueous humor and vitreous of patients with uveitis. Ophthalmology 1998; 105: 1659-1663.

4 Calder VL, Shaer B, Muhaya M, Mclauchlan M, Pearson RV, Jolly G et al. Increased CD4 + expression and decreased IL-10 in the anterior chamber in idiopathic uveitis. Invest Ophthalmol Vis Sci 1999; 40: 2019-2024.

5 Lacomba MS, Martin CM, Chamond RR, Galera JM, Omar M, Estevez EC. Aqueous and serum interferon gamma, interleukin (IL) 2, IL-4, and IL-10 in patients with uveitis. Arch Ophthalmol 2000; 118: 768-772.

6 Cushman M, Legault C, Barrett-Connor E, Stefanick ML, Kessler C, Judd HL et al. Effect of postmenopausal hormones on inflammation-sensitive proteins: the Postmenopausal Estrogen/Progestin Interventions (PEPI) Study. Circulation 1999; 100: 717-722.

7 Karakuzu A, Aktas A, Akcay F. Serum E-selectin and beta 2-microglobulin levels in Behcet's disease. J Int Med Res 2002; 30: 85-88.

8 Whitcup SM, Kozhich AT, Lobanoff M, Wolitzky BA, Chan CC. Blocking both E-selectin and P-selectin inhibits endotoxin-induced leukocyte infiltration into the eye. Clin Immunol Immunopathol 1997; 83: 45-52.

MA Elgohary

Ophthalmology Department

Essex County Hospital 
Lexden Road

Colchester CO3 3NB, UK

Correspondence: MA Elgohary

Tel: +447939274217

Fax: + 441206744742

E-mail: m.elgohary@doctors.org.uk

Eye (2005) 19, 1013-1015. doi:10.1038/sj.eye.6701706; published online 24 September 2004

Sir,

Reply to MA Elgohary, DYL Leung and DSC Lam

We would like to thank Dr Elgohary for the interest in and comments on our paper. ${ }^{1}$ The evidence for the relative importance of the immunomodulatory effects of oestrogen and progesterone remains inconclusive. In specifying the exact date of onset of acute uveitis, we were sensitive to the problem that there may be a short delay between immune 'trigger' and onset of symptoms. In view of this, it would be difficult to draw firm conclusions about the hormonal influence on particular days within the late phase of the menstrual cycle, at which time hormonal levels change precipitately. We can confirm that no patient was using concurrent oral steroid or immunosuppression at presentation.

We are also grateful for the comments on our paper ${ }^{1}$ by Leung and Lam. While we understand and appreciate the points made, we disagree with most. Almost all regularly menstruating women have excellent recall of the date of last menstrual period (LMP). Where there was doubt, the patient was not included in our study; there was therefore no recall bias on this parameter. We commented that it may be interesting to measure oestrogen and progesterone levels in such patients; however, the absence of these data does not affect the validity of our comments. Our proposition is not that uveitis commences at a particular hormone level, but that it may be precipitated by hormone withdrawal in the predisposed. It is not necessary to measure hormone levels to prove that a woman is in the late phase of the menstrual cycle, if the LMP date is known.

We strongly disagree that it is 'not unusual' for anterior uveitis to be relatively silent in the early phase of disease; all our patients had acute-onset anterior uveitis with clear memory of the date of onset of symptoms, and rapid attendance at our ophthalmic emergency department, usually within $24 \mathrm{~h}$. No patient had acuteon-chronic disease and no patient had recently discontinued topical steroid. Where there was doubt, the patient was excluded. We disagree that the aetiology of uveitis could affect presentation; while uveitis such as that related to juvenile idiopathic arthritis (JIA) can indeed be asymptomatic, that is not relevant to acuteonset symptomatic uveitis presenting in adulthood. We can confirm that no patient had JIA-related uveitis. We disagree that measurement of objective signs of severity might be a more reliable indicator of onset than reported symptoms; there is a wide variation in the severity of inflammation in those presenting with acute anterior uveitis; there is no evidence that this has a bearing on the duration of inflammation before presentation. On the contrary, it is not unknown for patients with recurrent anterior uveitis to be aware of recurrence (and to present with symptoms) before cells are detected in the anterior chamber. Finally, although the incidence of uveitis in the premenstrual phase did not quite reach statistical significance, the incidence in the whole postovulatory phase did; having carefully stated potential sources of error, we believe that our conclusions remain valid.

\section{References}

1 Sanghvi C, Aziz K, Jones NP. Uveitis and the menstrual cycle. Eye 2004; 18: 451-454.

C Sanghvi, K Aziz and NP Jones

Manchester Royal Eye Hospital

Oxford Road

Manchester M13 9WH, UK

Correspondence: NP Jones

Manchester Royal Eye Hospital

Oxford Road

Manchester M13 9WH, UK

Tel: + 441612765582

Fax: + 441612726618

E-mail: nicholas.jones@cmmc.nhs.uk

Eye (2005) 19, 1015. doi:10.1038/sj.eye.6701705;

published online 24 September 2004

Sir,

Eyelash poliosis in association with sarcoidosis

Sarcoidosis is a multisystem granulomatous disease. It frequently affects young adults, with a female 